ISSN: 0514-7336

DOI: http://dx.doi.org/10.14201/zephyrus201575165172

\title{
UNA NUEVA FIGURA ROJA EN LA CUEVA DE EL BUXU (CANGAS DE ONÍS, ASTURIAS)
}

\section{A new red figure in El Buxu Cave (Cangas de Onis, Asturias)}

\author{
Georges Sauvet \\ Centre de Recherche et d'Études pour l'Art Préhistorique (CREAP). Laboratoire TRACES (UMR 56-08). Université de \\ Toulouse-Jean-Jaurès. Maison de la Recherche. Allée Antonio Machado. F-31100 Toulouse-Cedex. Correo-e: georges. \\ sauvet@sfr.fr
}

Recepción: 22/11/2014; Revisión: 6/02/2015; Aceptación: 25/03/2015

BIBLID [0514-7336 (2015) LXXV, enero-junio; 165-172]

\begin{abstract}
Resumen: Esta nota tiene por objeto dar a conocer el descubrimiento fortuito de una figura zoomorfa roja en la cueva de El Buxu (Asturias). Se trata de la silueta de un cuadrúpedo con una pata por par interrumpida al nivel del corvejón, realizada por medio de un trazo grueso con tendencia al modelado parcial. Debido a la mala conservación, es difícil precisar la especie representada, que puede ser un cérvido o más probablemente un uro. A falta de un estudio completo que permitirá sin duda precisar la especie del animal y sus características estilísticas, presentamos unas fotografías y un simple croquis de lectura para dar una primera visión de la figura. El estilo antiguo de esta representación figurativa añade interés a los vestigios de pigmento rojo conocidos en la cueva asturiana en el contexto de las cuevas ante-magdalenienses de la Región Cantábrica.

Palabras clave: Arte rupestre; Región Cantábrica; figura zoomorfa; cuadrúpedo; pigmento rojo.
\end{abstract}

AвSTRACT: This brief article has for objet to present the fortuitous discovery of a red animal in El Buxu cave (Asturias). This is the silhouette of a quadruped with only one leg by pair without extremities drawn by a slightly modelling thick line. Due to the bad conservation, the represented species is difficult to identify: it might be a cervid or more probably an aurochs. This preliminary note presents only some photos and a sketch of reading, but a complete study will probably allow to identify the species of the animal and to precise its stylistic characteristics. This figurative representation with its stylistic features adds a new interest to the red traces already known in El Buxu cave in the context of the ante-Magdalenian caves of the Cantabrian Region.

Key words: Rock art; Cantabrian Region; zoomorphic figure; quadruped; red pigment.

(c) Universidad de Salamanca

Zephyrus, LXXV, enero-junio 2015, 165-172 


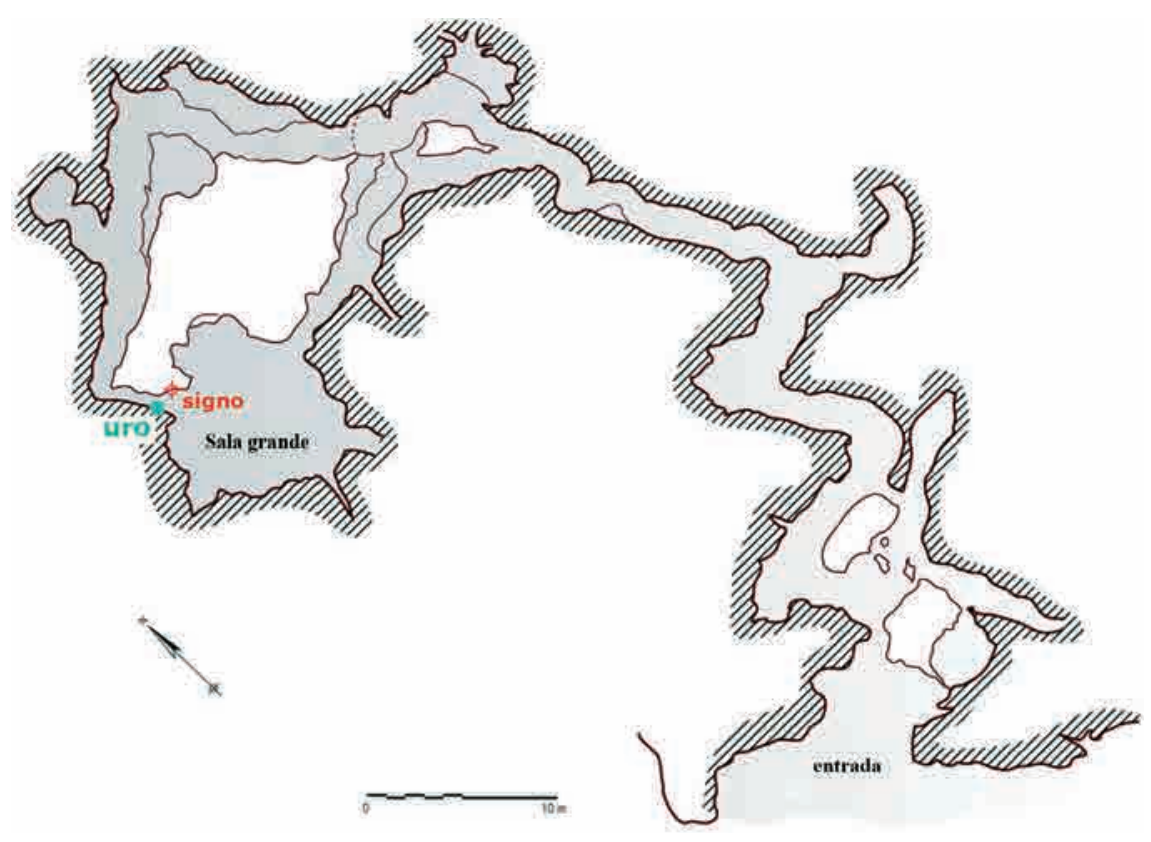

Fig. 1. Localización de la nueva figura en la 'Sala Grande' de la cueva de El Buxu. Plano modificado a partir de Hernanz et al., 2012. la cueva ${ }^{2}$. Pudimos examinar con toda tranquilidad este signo notable a la vez por su estructura y por su localización en un techo, sobre una especie de arcada entre dos pendientes de la bóveda. La sala donde se encuentra esta pintura, denominada 'Sala Grande' aunque los bloques que la obstruyen no dan la impresión de una sala de amplias dimensiones, se localiza al margen de las principales concentraciones de arte parietal de la cueva (Fig. 1). Durante esta visita, me llamó la atención una gran pared vertical situada frente al signo precedente, porque la parte baja de la misma presentaba una ancha superficie aparentemente manchada de rojo. Un examen visual atento mostró rápidamente que se podía discernir, en lo que parecían a priori simples vestigios de pigmento rojo, el contorno de un animal completo. La interpretación figurativa fue confirmada por las personas que nos acompańaban y en particular por Marta Soto.

El tratamiento de las fotografías digitales y la aplicación de filtros a través del plugin D-Stretch de ImageJ permitieron visualizar posteriormente esta figura con más nitidez, aunque una cierta ambigüedad subsiste en cuanto a la identidad de la especie representada.

En esta breve nota, no tenemos otro objetivo que dar a conocer esta nueva figura que aumenta el interés de las figuras rojas de El Buxu reveladas por M. Menéndez y otros vestigios rojos conocidos en la cueva desde los trabajos de Obermaier y el conde

2 Nuestro agradecimiento a Ignacio Alonso, director del Museo Arqueológico de Asturias, y a la Consejería de Cultura, que autorizó nuestra visita a la Sala Grande de El Buxu, que se encuentra fuera del recorrido turístico, y la toma de fotografías. Agradecemos igualmente a Marta Soto, que nos acompañó durante esta visita y compartió con nosotros el placer de un hallazgo fortuito. 


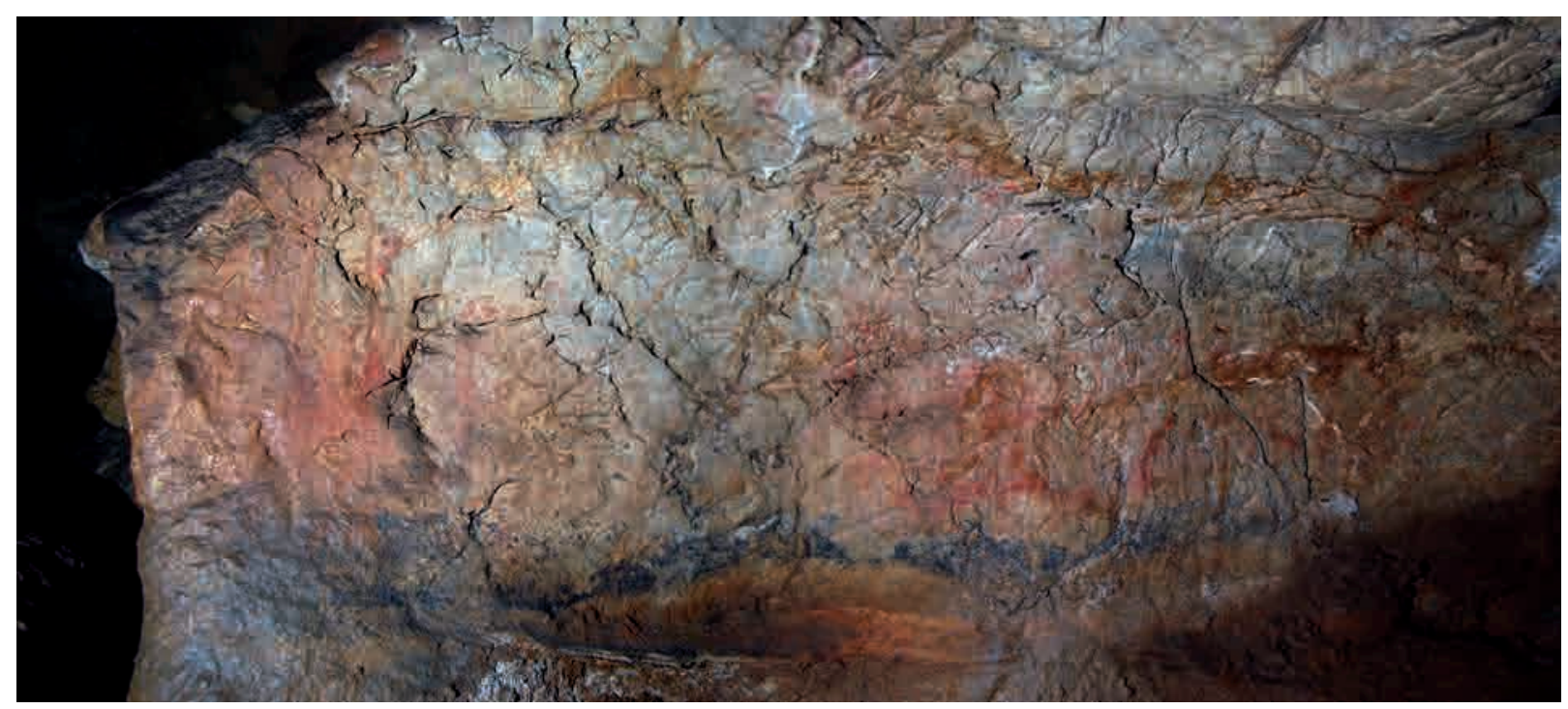

FIG. 2. Situación de la nueva figura en la 'Sala Grande' de la cueva de El Buxu.

de la Vega del Sella (1918) a los que cabe sumar diversas manchas y puntos señalados por M. Dams y L. Dams (1977). Sólo un estudio pormenorizado permitirá valorar plenamente las grafías rojas de El Buxu en el contexto iconográfico de las cuevas decoradas paleolíticas de Asturias.

\section{Descripción de la nueva figura}

La figura se localiza en una pared vertical bastante amplia limitada a la izquierda por un ángulo diedro y a la derecha por el arranque de una galería ascendente (Fig. 2). Se encuentra situada en un pequeño escalón calcificado, en la base de la pared. La figura es discernible a simple vista, pero las fotografías digitales, que otorgan un mejor contraste, facilitan la lectura.

Se trata de un cuadrúpedo de perfil izquierdo dibujado con un trazo ancho, en una actitud ligeramente descendente, según una línea de suelo que estaría más o menos materializada por una coloración más oscura de la roca (Fig. 3). Mide $80 \mathrm{~cm}$ del hocico a la grupa y los cuartos traseros están a $51 \mathrm{~cm}$ del suelo actual. La cabeza es sumamente alargada y puede corresponder a un cérvido o a un uro. Desgraciadamente, los elementos anatómicos que permitirían distinguir entre ambas especies son muy confusos. La parte superior de la cabeza es de lectura particularmente difícil pues toda la figura se encuentra rodeada de manchas de pigmento rojo. Únicamente se distingue una forma más o menos circular que interpretamos como el saliente del cráneo de donde partirían dos cuernos en perspectiva frontal, más que una cornamenta (Fig. 4). También el cuello grueso es un rasgo habitual de las representaciones de bóvidos, aunque algunos ciervos pueden presentar la misma masividad. Desafortunadamente, la cola, que permitiría resolver la duda, es muy poco legible, pero rastros de pigmento situados detrás de la nalga y paralelos a ella son compatibles con la hipótesis de una cola larga.

La pata anterior aprovecha con gran inteligencia un relieve de la pared que forma un bulto natural de algunos centímetros de anchura. Este relieve penetra profundamente dentro del cuerpo y está bordeado por una línea gruesa que discurre a lo largo de su borde posterior. Una segunda línea oblicua delimita el muslo y forma con la precedente un tipo de triángulo ventral. Parece poco probable que las extremidades anteriores y posteriores hayan sido representadas. Así el animal se define como una simple silueta con una pata por par sin extremidad (Fig. 5, n. ${ }^{\circ}$ 1). 

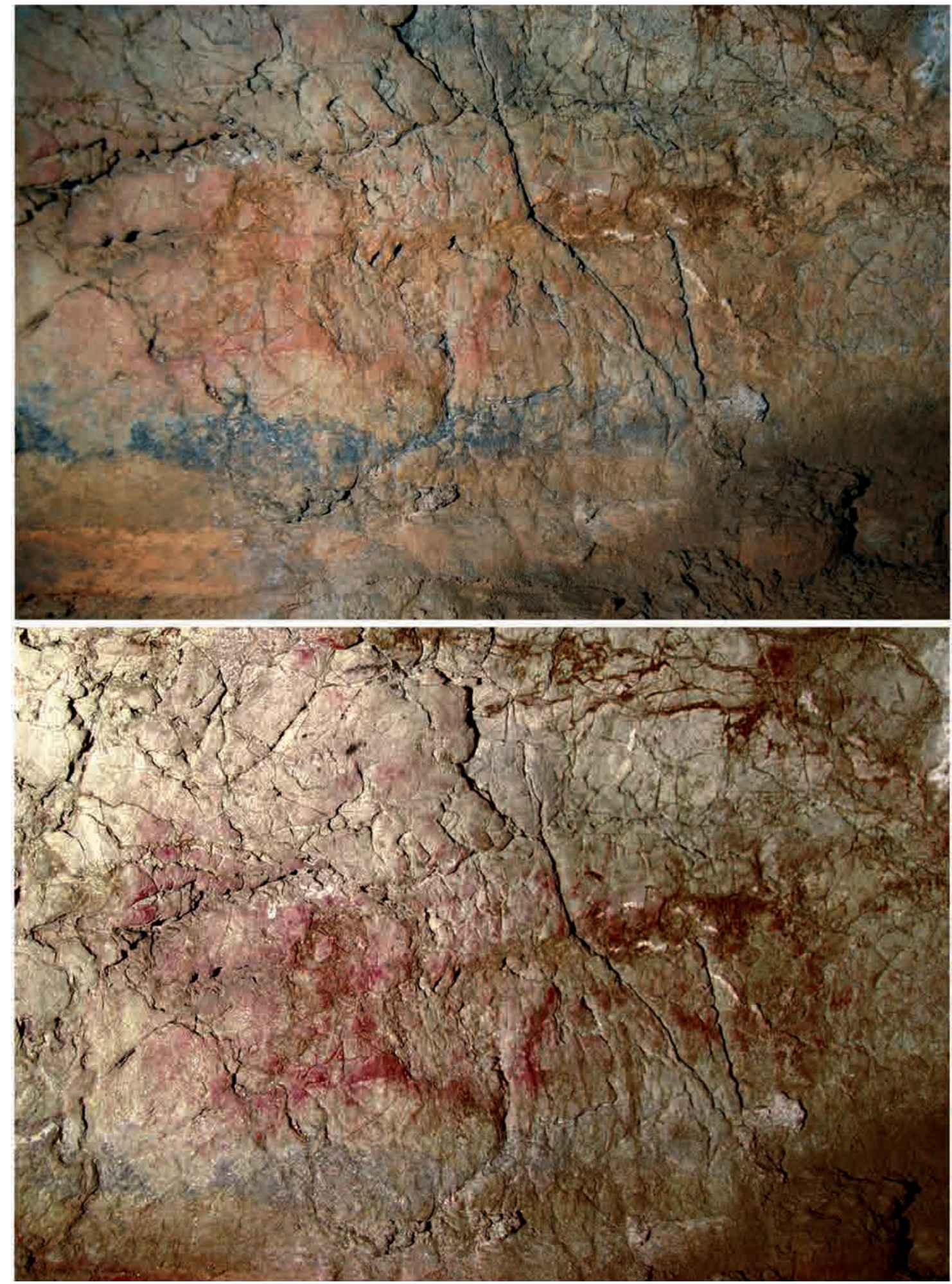

FIG. 3. Imagen del posible uro: A) fotografia no tratada; B) fotografía tratada con un filtro YRE de D-Stretch. 

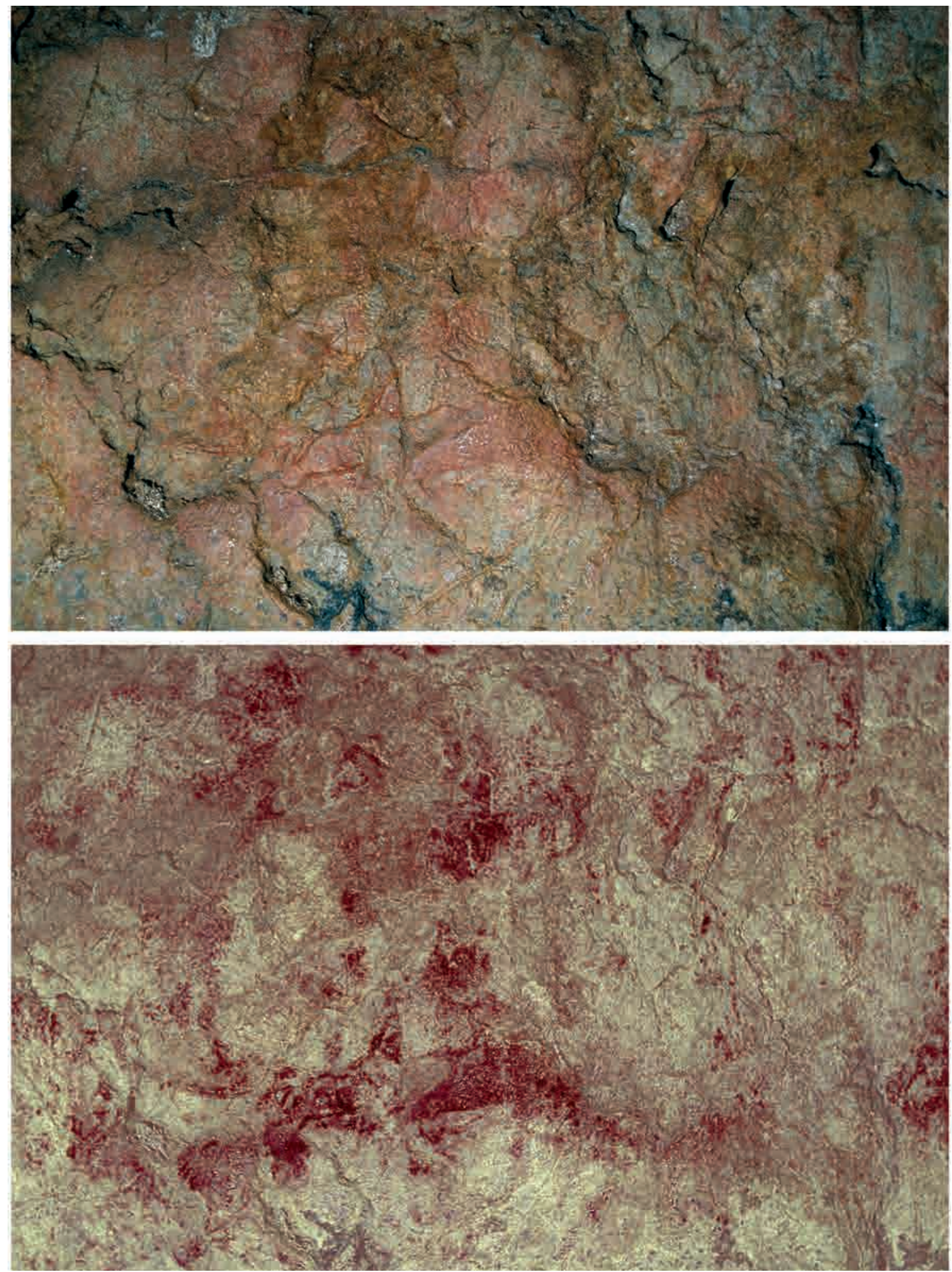

FIG. 4. Detalle de la cabeza: A) fotografía no tratada; B) fotografía tratada con un filtro YRE de D-Stretch. 
El trazo de contorno presenta varios espesamientos principalmente en el cuello y en el raquis, que se pueden interpretar como un intento de modelado parcial. En el muslo se aprecia un esbozo de relleno. Numerosos vestigios de pigmento rojo son visibles no sólo dentro del cuerpo del animal, sino también sobre toda su periferia. Estos rastros se concentran por encima de la línea dorsal y de la cabeza, pero se extienden más allá formando un halo difuso alrededor del animal.

\section{El contexto de las figuras rojas cantábricas}

Las figuras rojas de la Región Cantábrica han sido objeto de varios trabajos recientes ${ }^{3}$ (Garate, 2006; Hernando, 2014). No iniciaremos aquí una discusión pormenorizada respecto a su cronología, contentándonos con anotar que hay un consenso en otorgarles una atribución cultural relativamente antigua que oscila entre Gravetiense y Solutrense superior. La mayoría de las figuras son ciervas realizadas con la técnica punteada. Los ciervos pintados pertenecientes al periodo "ante-magdaleniense" computados por C. Hernando (2014) son 35 y los uros pintados, 20. Los uros presentan una gran variabilidad formal que va desde siluetas en trazo simple a figuras modeladas en tinta plana más o menos completa (Fig. 5). Algunas tienen sólo un miembro por par, la mayoría de las veces sin extremidad (La Pasiega A, Fig. 5, n.o 2), mientras que otros tienen dos miembros por par, figurados en perspectiva natural y provistos de cascos (Trescalabres, Fig. 5 , n. $^{\circ}$ 3). Entre estas representaciones, la que presenta las similitudes más notables con la figura de El Buxu es sin duda una de La Pasiega situada sobre una pendiente de la bóveda hacia el fondo de la galería A (Fig. 5, n. ${ }^{\circ}$ 2).

Uno de los rasgos comunes a un buen número de estas representaciones de uros es su hocico muy largo y fino, como en el caso de una cabeza

\footnotetext{
${ }^{3}$ Garate, D.: Análisis y caracterización de los conjuntos parietales con grafías zoomorfas punteadas. Una expresión pictórica propia del Paleolitico superior cantábrico. Tesis doctoral inédita presentada en 2006 en la Univ. de Santander; Hernando, C.: La sociedad a través del arte: las tradiciones gráficas premagdalenienses en la Región Cantábrica. Tesis doctoral inédita presentada en 2014 en la Univ. de Salamanca.
}

del Castillo (Fig. 5, n.o 6). Es interesante anotar que no es una característica únicamente cantábrica, ya que la encontramos casi idéntica en la cueva de La Tête-du-Lion (Ardèche) en un contexto cronológico del Solutrense antiguo (Fig. 5, n. ${ }^{\circ}$ 8). Este alargamiento excesivo de la cabeza es un carácter estilístico que no debe ser confundido en ningún caso con un rasgo que indique que se trata de una especie distinta. Animales que presentan esta particularidad han sido identificados a veces en la literatura como Bos longifrons (Breuil, 1952: 43), aunque esta especie no existía, al menos durante el Pleistoceno (Koby, 1954).

Sin embargo, debemos mencionar que el espesor del cuello característico de la figura de El Buxu se observa a veces en representaciones de ciervos como en el caso de Salitre (Fig. 5, n. ${ }^{\circ}$ 10), lo que mantiene una cierta duda cuanto a su identificación específica. En cualquier caso, la nueva representación animal de El Buxu se incorpora de lleno en el corpus de figuras ante-magdalenienses. Muchos de los sitios que hemos mencionado a título de comparación poseen restos de ocupación del Solutrense superior (La Pasiega, Trescalabres, La Haza), como es igualmente el caso de esta cueva (Menéndez, 1984), pero nos parece prematuro deducir un argumento cronológico concreto de este hecho.

\section{Conclusión}

La nueva representación figurativa de El Buxu que interpretamos preferentemente y a título provisional como un uro se integra sin dificultad en el conjunto de figuras rojas de la fase antigua del Paleolítico superior, tanto por su técnica a base de trazos lineales rojos ligeramente modelados como por sus características estilísticas. Hasta el momento, las únicas grafías rojas catalogadas en esta cueva eran signos: un signo en forma de $\mathrm{E}$ ya descrito en la monografía inicial (Obermaier y Vega del Sella, 1918), numerosos puntos y rastros diversos repartidos en toda la caverna (Dams, 1977) y el signo recientemente interpretado por Menéndez y García (2014) como un signo femenino. La incorporación de un animal de características estilísticas claras corrobora la existencia de un arte parietal premagdaleniense (Solutrense o más antiguo) en la cueva. Asimismo, esta figura amplía el conjunto de figuras 


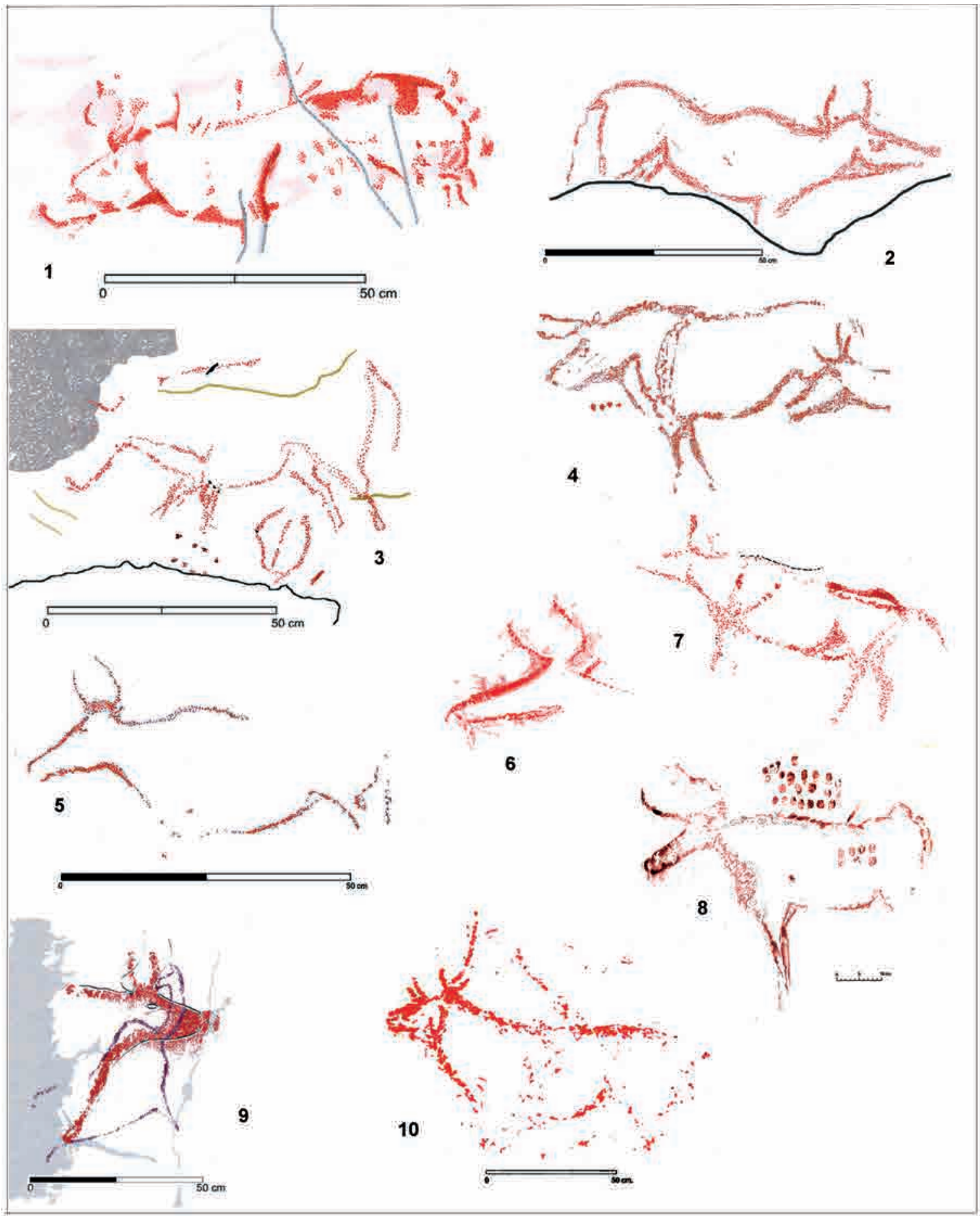

Fig. 5. Representaciones de uros y ciervos ante-magdalenienses: 1) croquis de lectura del probable uro de El Buxu (Asturias); 2 y 4) La Pasiega A (Cantabria); 3) Trecalabres (Asturias); 5) La Pasiega C (Cantabria); 6) El Castillo (Cantabria); 7) La Haza (Cantabria); 8) La Tête-du-Lion (Ardèche); 9. La Garma (Cantabria); 10) El Salitre (Cantabria). N. os 2-7 y 9 según Garate, 2006; n. 8 según Ayroles en Combier, 1984; n. 10 según González Sainz et al., 2009. 
rojas del oriente de Asturias, ya bien nutrido (El Pindal, Llonín, Cordoveganes, Trescalabres, Tito Bustillo, Les Pedroses, etc.), sin olvidar el reciente descubrimiento de Pruneda (Onís) a unos $10 \mathrm{~km}$ de El Buxu, en el mismo valle del río Güeña. Futuras investigaciones permitirán asegurar la identificación específica de esta representación. En particular, sería de sumo interés un análisis de pigmento por espectroscopia Raman como el ya practicado para cuatro muestras de pigmento rojo de la cueva (Hernanz et al., 2012), que permitiría la comparación con el signo de la misma zona que tiene la particularidad de contener manganeso y fósforo como elementos secundarios.

\section{Bibliografía}

Brevil, H. (1952): Quatre cents siècles d'art pariétal. Montignac: Centre d'Études et Documentation Préhistoriques.

Combier, J. (1984): "Grotte de la Tête-du-Lion". En Leroi-Gourhan, A.: L'art des cavernes. Atlas des grottes ornées paléolithiques françaises. Paris: Ministère de la Culture-Imprimerie Nationale, pp. 595-599.

DAms, M. y DAms, L. (1977): "Topografía e iconografía complementarias de la caverna del Buxu (Asturias)", Trabajos de Prehistoria, 34, pp. 327-330.
González Sainz, C.; Garate, D. y Eguizabal, J. (2009): "Contribución al conocimiento del arte parietal paleolítico de la cueva de El Salitre (Miera, Cantabria)”, Sautuola, Xv, pp. 439-451.

Hernanz, A.; Gavira-Vallejo, J. M.; Ruiz-López, J. F.; Martin, S.; Maroto-Valiente, A.; Balbín-Behrmann, R.; Menéndez, M. y Alcolea-GonzáLEZ, J. J. (2012): “Spectroscopy of Palaeolithic rock paintings from the Tito Bustillo and El Buxu Caves, Asturias, Spain”, J. Raman Spectroscopy, 43 (11), pp. 1644-1650. http://dx.doi.org/10.1002/jrs.3145

Koвy, F. E. (1954): "Y a-t-il eu, à Lascaux, un 'Bos longifrons'?", Bulletin de la Société Préhistorique Française, t. 51, n. ${ }^{\text {os } 9-10, ~ p p . ~ 434-441 . ~}$

Menéndez, M. (1984): "La cueva del Buxu. Estudio del yacimiento arqueológico y de las manifestaciones artísticas", Boletín del Instituto de Estudios Asturianos, 111, pp. 143-185.

Menéndez, M. y García, B. (2014): “El nuevo horizonte de pinturas rojas de la Cueva del Buxu. Asturias. España”. En Corchón, M. S. y Menéndez, M. (eds.): Cien años de arte rupestre paleolitico. Salamanca: Univ. de Salamanca, pp. 63-73.

Obermaier, H. y Conde de la Vega del Sella (1918): La Cueva del Buxu (Asturias). Memoria, 20. Madrid: Comisión de Investigaciones Paleontológicas y Prehistóricas. 